\title{
Improving mesh generation in finite element analysis for functional morphology approaches
}

\author{
Jordi MARCÉ-NOGUÉ ${ }^{1,2^{*}}$, Josep FORTUNY ${ }^{2,1}$, Lluís GIL \& Montserrat SÁNCHEZ ${ }^{1}$
}

${ }^{1}$ Departament de Resistència de Materials i Estructures a l’Enginyeria, Escola Tècnica Superior d'Enginyeria Industrial i Aeronàutica
de Terrassa, Universitat Politècnica de Catalunya, C/ Colom 11,08222 Terrassa, Barcelona, Spain; jordi.marce@upc.edu;
1luis.gil@upc.edu; montserrat.sanchez@upc.edu
22 Institut Català de Paleontologia, Edifici ICP, Universitat Autònoma de Barcelona, Cedanyola del Vallès, Spain;
josep.fortuny@icp.cat

* Corresponding author

Marcé-Nogué, J., Fortuny, J., Gil, L. \& Sánchez, M. 2015. Improving mesh generation in finite element analysis for functional morphology approaches. [Mejoras en la generación de mallas de elementos finitos en análisis de morfología funcional]. Spanish Journal of Palaeontology, 30 (1), 117-132.

\begin{abstract}
Finite Element Analysis (FEA) is a powerful tool for functional morphology purposes. The accuracy of the final results depends on the mesh generation and the quality of the mesh. This is especially important in vertebrates as they present a complex biological structure, implying a complex geometry and, consequently, mesh generation should be performed with a consistent criterion. The aim of this paper is to discuss different ways to create a mesh of a vertebrate structure with different mesh generation methods and give recommendations on how to generate an efficient mesh without exceeding computational limits. Topics such as quality of the mesh, suitability of the mesh and reliability of the mesh are introduced to help the generation of the mesh. In this case, the use of convergence procedures assures the results of the computational solution and can be a good solution for the vertebrate models. The skull of a Chinese giant salamander (Andrias davidianus) is used as a case study.
\end{abstract}

\section{RESUMEN}

El Análisis de Elementos Finitos (FEA) es una poderosa herramienta para los estudios en morfología funcional. La exactitud de los resultados finales depende de la generación de la malla y la calidad de ésta. Esto es especialmente importante en los vertebrados, ya que presentan una estructura biológica compleja, lo que implica una geometría compleja y, en consecuencia, la generación de la malla debe realizarse con un criterio coherente. El objetivo de este trabajo es discutir diferentes formas de crear una malla de una estructura de vertebrado con diferentes métodos de generación de malla y dar recomendaciones sobre cómo generar una malla eficiente sin sobrepasar los límites computacionales. Temas tales como la calidad de la malla, la adecuación de la malla y la fiabilidad de la malla se introducen para ayudar a la generación de ésta. El uso de procedimientos de convergencia en la generación de la malla puede asegurar los resultados obtenidos en la solución computacional y puede ser una buena solución 
Keywords: Finite Element Method, computational analysis, mesh generation, vertebrate palaeontology, functional morphology. a adoptar en los modelos de vertebrados. Cómo ejemplo se estudia un cráneo de una salamandra gigante de China (Andrias davidianus).

Palabras clave: Método de los Elementos Finitos, Análisis computacional, generación de malla, paleontología de vertebrados, morfología funcional.

\section{INTRODUCTION}

Finite Element Analysis (FEA) (Bathe, 1996) is a powerful tool in vertebrate palaeontology. Its use has been conducted particularly in mammals and reptiles in the last ten years and provides new insights to explore the function, morphological evolution, particular adaptation and constraints of biological structures (Rayfield et al., 2001; Strait et al., 2005; McHenry et al., 2007; Jasinoski et al., 2009; Moazen et al., 2009, Dumont et al., 2011; Bright \& Rayfield, 2011a; Fortuny et al., 2011, 2012 amongst others). The aim of these studies is to test how well-adapted an organism is for a particular function. Nevertheless, these predictions are not always plausible as demonstrated by neontological work (Lauder, 1995).

The application of FEA in the study of the vertebrates remained unexplored until the late 90's due to the complexity of biological geometry and its high computational cost. Fortunately, the rapidly falling prices of computers and the rapid increase in computational capacity over the years facilitated the use of FEA in estimating the performance of vertebrate skeletal and soft tissues. Vertebrate palaeontologists also found non-invasive techniques, such as computed tomography (CT), a useful tool to generate accurate three-dimensional images of living structures; CT scanning enables the possibility of performing these kinds of studies at present (see Rayfield, 2007 for a review).

It is widely known that several inputs affect the creation of a suitable biological FEA model to be solved in a FEA software Package or a FEA code (Rayfield, 2007). The accuracy of the results obtained depends on the assumptions implicit in the model: geometrical simplifications of the bone and sutures, elastic assumptions in material properties, appropriate constraints and applied loads as well as the procedures and methods to generate the mesh.

Due to the complexity of the biological structure's geometry, it should be remarked that the generation of the Finite Element mesh and the techniques to generate it is a key topic for the accuracy of the final results obtained with FE methodologies. Nevertheless, this question has not been widely discussed in the paleontological literature.

In this paper we focus on mesh generation using FEM in vertebrate palaeontology to solve biomechanical problems using only the elasticity equations. Heat transfer, fluid dynamics, etc. are not considered.

The aim of this paper is to discuss different ways to create and optimize a mesh with a consistent criterion for vertebrate structures and to recommend how to generate a good-quality mesh without exceeding the capabilities of the computer as well as other suggestions to improve the mesh generation. We have analysed the mesh obtained from the skull of a Chinese giant salamander (Andrias davidianus), as an example to illustrate the different procedures and recommendations that can be used to generate an optimal mesh.

\section{CONSIDERATIONS USING FINITE ELEMENT MESHES}

\subsection{Considerations about mesh generation}

As shown in Table 1 with the number of elements of several models listed, the mesh of three-dimensional vertebrate structures implies fine meshes and the possibility to generate a mesh with more than one million of elements.

The size of the mathematical system of equations solved by the computer is directly related to the total number of nodes of the domain. It should be noted that the number of elements is not the best indicator of the mathematical equation system size (as well as to use as indicator of the size of the mesh). The number of nodes differs depending on the type of element used (see Supplementary Information for further details in type elements). Consequently, the time required for the computer to generate the mesh and obtain a solution differs significantly.

As suggested by previous works (Richmond et al., 2005; Rayfield, 2007; Wroe et al., 2007a) the first recommended step is to make a previous analysis with a coarse mesh with few elements to solve, as a first approximation, to explore the physical problem of the case study. In this work, coarse meshes and fine meshes were generated (see below) from the whole skull and both results were compared. The meshes were generated using two different methods. 
Table 1. Examples of number of elements in a 3D FEA of some palaeontological studies.

\begin{tabular}{llll}
\hline Year & \multicolumn{1}{c}{ Reference } & \multicolumn{1}{c}{ Skull Model } & \multicolumn{1}{c}{ Elements } \\
\hline 2001 & Rayfield et al., 2001 & Allosaurus fragilis & 146,398 \\
2005 & Richmond et al., 2005 & Macaca fascicularis & 290,639 \\
2005 & Strait et al., 2005 & Macaca fascicularis & 311,057 \\
2007 & Wroe et al., 2007a & Canis lupus dingo & 887,281 \\
2007 & Wroe et al., 2007a & Thylacinus cynocephalus & 138,2216 \\
2007 & Grosse et al., 2007 & Artibeus jamaicensis & 113,3096 \\
2007 & McHenry et al., 2007 & Smilodon fatalis & $\approx 1,800,000$ \\
2007 & Wroe et al., 2007b & Pan troglodites & $3,023,365$ \\
2008 & Moazen et al., 2009 & Uromastyx hardwickii & $\approx 215,000$ \\
2009 & Jasinoski et al., 2009 & Oudenodon bainii & 462,447 \\
2010 & Jasinoski et al., 2010 & Lystrosaurus declivis & $1,087,376$ \\
2011 & Dumont et al., 2011 & Callithrix jacchus & $1,136,737$ \\
2011 & Dumont et al., 2011 & Saguinus fuscicollis & $1,248,605$ \\
2011 & Bright \& Rayfield, 2011a & Sus scrofa & $1,749,149$ \\
\hline
\end{tabular}

The first method is called here uniform mesh and implies the use of Delaunay method (Peraire et al., 1987) or Advancing front method (Lohner \& Parickh, 1988) to generate an uniform tetrahedral mesh. A surface mesh of tetrahedrons on the external surface of the domain is generated using one of these methods which respect all faces and edges in the geometry creating a uniform size of the elements in the whole mesh. The volume mesh is then created from the surface mesh via an algorithm that generates prism inflation layers into the tetra volume mesh created (see Supplementary Information for further details).

In the second method (called here adaptive mesh) a tetrahedral volume mesh is generated using a spatial subdivision algorithm as, for example, quadtree method (see supplementary information for further details). This algorithm creates an adaptive mesh which ensures refinement of the mesh where necessary, but maintains larger elements where possible. This method does not respect the faces and edges in the geometry unless a boundary condition is applied. Although for biological or palaeobiological models it is often not the goal of the analysis, when the interest is to find the exact values of stress or strain is highly recommended to use a local adaptive mesh instead of generating an adaptive mesh for the entire domain because the accuracy of these results will be better.

This can be done by starting with a very coarse mesh (for the whole domain) and later generating a convergence test to only refine the areas of interest in order to generate an adaptive fine mesh in these areas. Convergence test records a result within a specific location against some measure of mesh density as the refinement iteration. The convergence is achieved when the difference between the two last iterations is less than a certain error threshold (see below and Supplementary Information for further details about convergence tests).

Although the influence of element size in the recorded values of strain, stress and deformation has been studied successfully in regular meshes (Bright \& Rayfield, 2011b), herein a step-beyond procedure, such as the local converged adaptive mesh, will be tested and discussed.

\subsection{Considerations of the results}

In FE Analysis of vertebrate structures, distribution and maximum values of equivalent von Misses stresses, principal stresses, strains and total deformations are usually recorded to compare or study their behaviour under the effect of the loads and constraints defined for each problem (Kupczik, 2008).

Furthermore, mesh convergence tests are a useful tool to look for the optimal solution to ensure the best result with the coarsest possible mesh. But convergence tests can generate artificial noise with divergence stresses in some points where the record value follows a vertical asymptotic with tendency to increase the value towards infinity when the mesh is refined (see Supplementary Information for convergence/divergence mathematical concepts). This artificial noise is a numerical singularity and is well-known in the field of the FEA (Morris, 2008).

This singularity could be a consequence of simply supported boundary conditions or a perfect square within the geometry. A simply supported condition is an idealistic assumption. This condition creates a numerical effect that leads to the stress increasing without limit when the mesh is refined for both 2D and 3D elements. In similar way, the perfect square condition is also an idealistic assumption where a corner with zero radius creates a stress concentration that also leads the value to increase without limit when the mesh is refined. To avoid this artificial noise, it is not recommended to record the maximum stresses or strains values in the whole domain because maximum values could coincide with artificial noise. As some other authors warned about these considerations (Rayfield, 2007), it may be difficult to assess the peak stresses of the model in the whole domain. However, analysis in a particular area of interest can avoid this problem. Following this procedure, the areas of interest are suitable for convergence, and omit the singularity provided by the maximum peak stresses or strains values where artificial noise could appear.

Another option to avoid artificial noise produced by the singularity in the simply supported boundary condition is to use a very coarse mesh in this area. The low accuracy of the coarse mesh prevents the artificial noise being recorded and moreover, in spite of the mesh, the stiffness of the model is not modified and the deformation values can still be recorded.

On the other hand, using FE methodologies is usually necessary to compare the results obtained from different taxa analysed. Some authors (Strait et al., 2005; Grosse et al., 2007; Pierce et al., 2009; Fletcher et al., 2010; Jasinoski et al., 2010) recorded the maximum stress and 
strain values at the same selected points for each model they wanted to compare. This procedure could be very useful especially when the aim is a validation study in which in vivo experimental bone strain data are collected in the same locations (Strait et al., 2005; Bright \& Rayfield, 2011a). In this case, the values obtained with FEA can be compared with the values of the experimental test.

Nevertheless, if the aim of study is not intended to visualize absolute stress and strain of the vertebrate structure, but instead is an attempt to generate useful comparative models, a general stress pattern may be accepted. Thus, a qualitative analysis of the general stress patterns could be made when the aim is to observe the relative similarities and differences in the mechanical behaviour instead of recording the exact values of stress.

The only analysis that can avoid all these suggestions and recommendations about the mesh and how to avoid artificial noise is when the case study is to obtain the reaction values or deformation fields (Clausen et al., 2008). This type of analysis does not generate artificial noise and any mesh can be used to solve these models (an efficient mesh could be a very coarse one).

\section{CASE STUDY}

\subsection{Analysed specimen}

The specimen used is the skull of the Chinese giant salamander (Andrias davidianus). This cranial material (MZB - 2001-0961-B) housed in the Museu de Ciencies Naturals de Barcelona corresponds to an adult specimen. The Andrias genus is represented by two living species of giant salamanders and includes the largest extant amphibians.

\subsection{Computed tomography}

The skull of Andrias davidianus was scanned with a medical CT Siemens ${ }^{\circledR}$ Sensations-16, at $140 \mathrm{kV}$ and 150 mAs giving an output of $512 \times 512$ pixels per slice. The pixel size and the inter-slice space were $0.586 \mathrm{~mm}$ and $0.1 \mathrm{~mm}$, respectively. CT data were imported to Mimics ${ }^{\circledR}$ software to obtain a 3-D model of the skull.

\subsection{Finite Element Analysis procedures}

The solving procedure and the mesh generation were performed using the automatic algorithms available in the FEA package ANSYS 12.1 operating under Windows XP (32-bit system) on a Intel ${ }^{\circledR}$ Core $^{\text {TM}} 2$ Quad, $800 \mathrm{MHz}$ with 4 GB of RAM. Elastic properties were assumed for the cortical bone, using the following values: E (Young's modulus of Elasticity): $6.65 \mathrm{GPa}$ and v (Poisson's ratio) 0.35 . These values are hypothetical and were only used to test the generated mesh. In any case, our purpose is to present procedures and methods to obtain an optimal mesh for the Chinese giant salamander (Andrias davidianus) and no biological implications could be inferred. Other research cover this issue, discussing the functional morphology of this giant amphibian (Fortuny et al., 2015).

In this case study, the quality of the mesh, the elapsed time and the reliability of the results obtained were studied in 5 different meshes: a coarse mesh and a fine mesh created with the two methods described above, and a local converged mesh. The local convergence mesh has been generated starting from a very coarse Uniform mesh. It is done in this way because a convergence procedure is not allowed in the Adaptive mesh. To compare the values of stress at different points on the skull, three middle lines are marked in the model: the first in the upper part of the skull, the second in the lower part of the skull and the third in the inner part of the skull (Fig. 1). The values of the stress results were recorded in these lines. Far from the singularities in the fixed points, these lines are located in a good place to test the values of stress in the different meshes and in the convergence procedure.

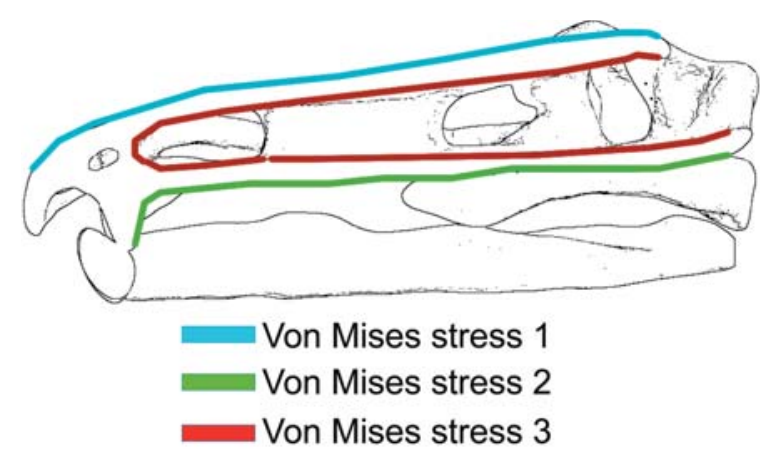

Figure 1. First line in the upper part, second in the lower part and third line in the inner part of the skull where the Maximum Von Mises stress are captured in the four meshes.

\subsection{Loading conditions of the model}

The biomechanics of $A$. davidianus can be modelled as a system of levers, as the skull and jaws form a single lever system. In this simplified attempt, the model was fixed by the occipital condyles and was loaded from the pterygoid and the articular-coronoid (Meckel cartilage area) to simulate the skull-lower jaw movement during open-close of the mouth, as the movement of the skeletal 
levers is accomplished by the contraction of the associated musculature. Herein, the hyobranchial skeleton was omitted as it is out of the scope of the present article. In the case of the lower jaws, the depressor mandibulae and the levator mandibulae act both as antagonists when they open or close the mouth. (Deban \& Wake, 2000) (Fig. 2).

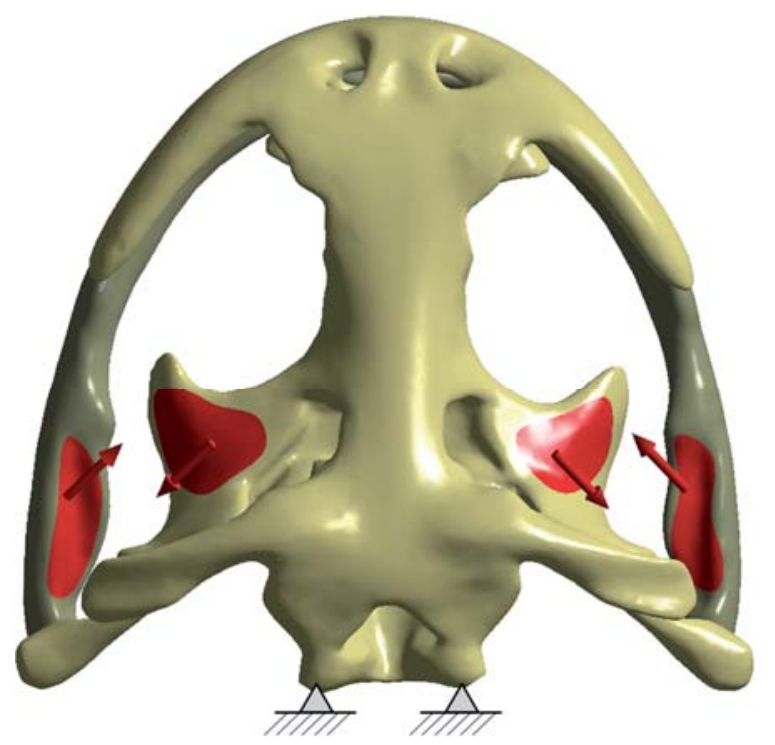

Figure 2. The model of the Chinese giant salamander (Andrias davidianus) simply loaded by the pterygoid (skull) and the articular-coronoid (Meckel's cartilage area) (jaw).

\section{RESULTS}

Firstly, we generated 4 different meshes; using the two methods described above, we created a coarse tetrahedral mesh and a fine tetrahedral mesh for each method of generation (uniform and adaptive meshes).

Table 2 shows the different meshes considered in this work. By changing an element size indication for the whole mesh, the FEA package generates a fine mesh with a high number of nodes and elements instead of a coarse mesh. Table 2 also indicates in seconds the elapsed time spent to create the mesh and the quality indicators corresponding to the aspect ratio and the skewness of the mesh obtained by each different mesh. Quality Indicators inform on the quality of the mesh, such as indicators for measuring a specific item or the whole shape of the mesh (see Supplementary Information for further details about quality indicators).

Secondly, a local convergence procedure was performed in the Chinese giant salamander model to record the stress maximum values of von Mises in the three middle lines previously mentioned (Fig. 1). An initial coarse mesh is needed to start the procedure before obtaining the final converged mesh (mesh 5, Table 2). The elapsed time used to generate the mesh in the local convergence procedure includes the elapsed time spent in solving the equations and obtaining the results. Herein, an automatic convergence procedure is used (see Supplementary Information for further details about automatic convergence).

The results obtained for the 5 different meshes can be observed in Figure 3, where each one shows the deformation distribution and the von Mises stress distribution. The higher values obtained in the three middle lines of Figure 1 are captured for the five different meshes in Table 3. This table also shows the elapsed time spent to solve the mesh in seconds.

\section{DISCUSSION}

As previously mentioned, when the size of the mesh is smaller, the result will be more accurate. Consequently, reducing the size of the elements of the mesh could be a good option to improve the results, even though it increases the computational cost of the problem. Subsequently, using tools as a convergence test are a good option to find the optimal mesh size. If the size of the elements is reduced, then the quality of the mesh will also improve.

Table 2. Different meshes considering the method in the mesh generation with nodes and elements of the model, elapsed time and quality indicators. *Mesh 5 includes the mesh generation and the solve Elapsed Time.

\begin{tabular}{rlccccccc}
\hline Case & \multicolumn{1}{c}{$\begin{array}{c}\text { Mesh } \\
\text { Generation } \\
\text { Method }\end{array}$} & Nodes & Elements & $\begin{array}{c}\text { Elapsed } \\
\text { Time [s] }\end{array}$ & Average & $\begin{array}{c}\text { Aspect ratio } \\
\text { Standard } \\
\text { deviation }\end{array}$ & \multicolumn{2}{c}{ Skewness } \\
Average & $\begin{array}{c}\text { Standard } \\
\text { deviation }\end{array}$ \\
\hline Mesh 1 & $\begin{array}{l}\text { Coarse } \\
\text { Uniform }\end{array}$ & 31,209 & 16,527 & 40 & 4.2585 & 16.4060 & 0.5880 & 0.2477 \\
Mesh 2 & Fine Uniform & 426,165 & 248,183 & 170 & 2.3105 & 0.8369 & 0.4190 & 0.1974 \\
Mesh 3 & Coarse Adaptive & 31,727 & 17,743 & 210 & 2.1803 & 0.7669 & 0.3473 & 0.1616 \\
Mesh 4 & Fine Adaptive & 531,677 & 359,631 & 380 & 1.8581 & 0.4366 & 0.2213 & 0.1541 \\
Mesh 5 & $\begin{array}{l}\text { Final converged } \\
\text { mesh }\end{array}$ & 94,464 & 59,676 & $265^{*}$ & 2.1732 & 0.8765 & 0.3137 & 0.1243 \\
\hline
\end{tabular}



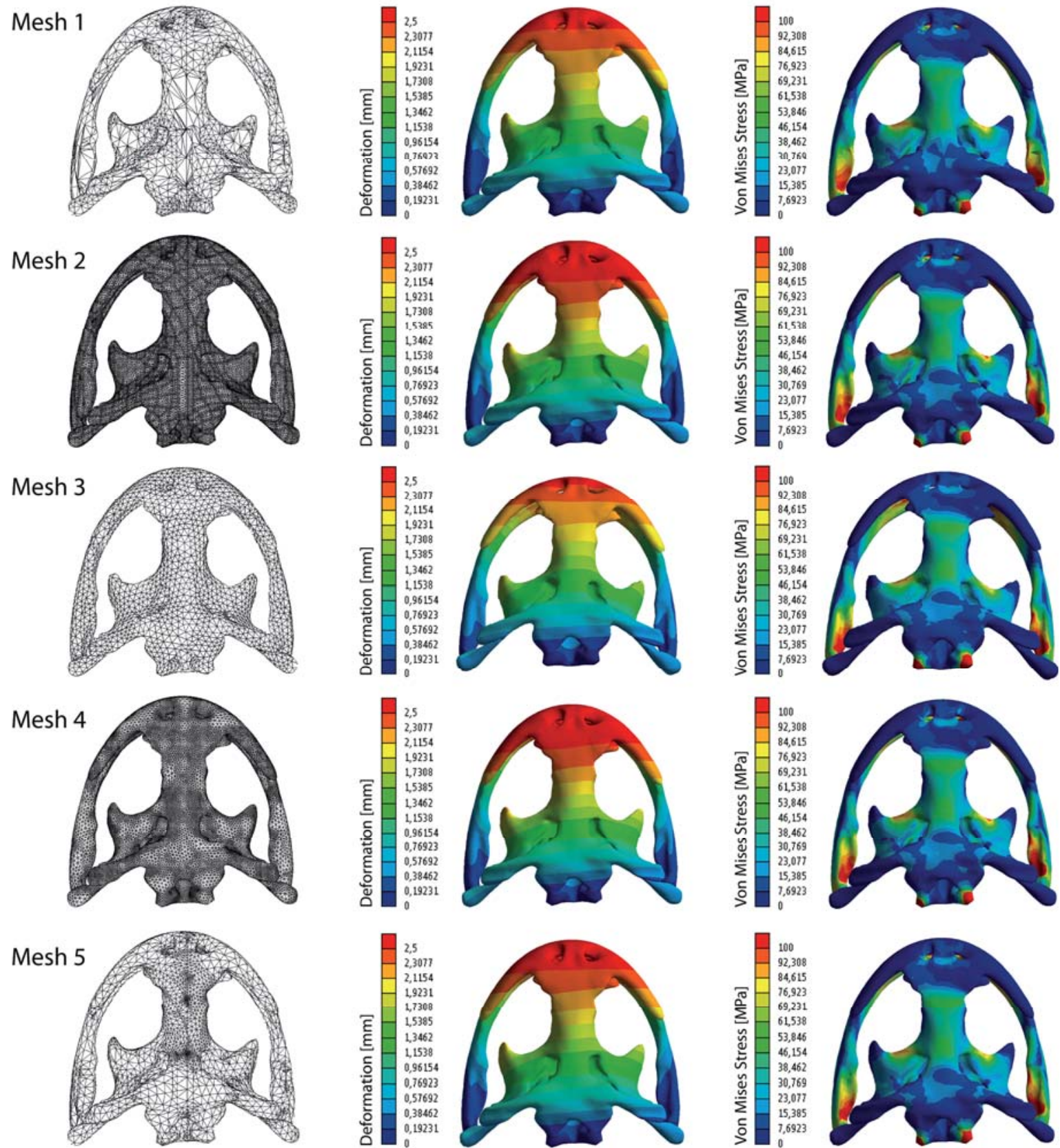

Figure 3. The 5 different meshes with the deformation distribution and the Von Mises stress distribution obtained. Mesh 1: Coarse Uniform; mesh 2: Fine Uniform; mesh 3: Coarse Adaptive Mesh; mesh 4: Fine Adaptive; mesh 5: final Converged Mesh.

\subsection{Quality of the mesh}

The quality of the mesh evaluates the precision when the element integrates the equations in the domain of the element to obtain the results of the mathematical problem by the Finite Element Method.

The natural tendency observed in all the meshes generated is to increase the quality when the overall element size of the mesh is small (ideally, the mesh aspect ratio must be 1 and the mesh skewness must be 0). This means that the quality indicators tend to reach the most optimum mesh. Otherwise, the Adaptive method generates meshes with better quality indicators because the mesh is better suited to the irregular geometry of the skull.

In our case, the quality indicators demonstrate that Fine Adaptive mesh (mesh 4) (see Table 2) improves the quality indicators more than $50 \%$ comparing to the uniform mesh with a coarse mesh. In other words, it is not a recommended procedure to create a mesh in which all the elements have the same size. Even if the size of the elements is small, the mesh efficiency (elapsed time vs. quality) is not good. Table 2 also shows that, when 
a convergence procedure is applied in a domain or in a partial domain (mesh 5), the quality of the mesh will be automatically improved in the same way as the elements of the mesh reduce its size.

\subsection{Suitability of the mesh}

The suitability of the mesh evaluates how the distribution of the different sizes in the mesh is well adapted to the changes in the stress gradients. For example, an important point to take into account is that in the regions of the domain with constant stress pattern (without large stress gradients), a coarse mesh could be used while, in the areas with large stress gradient, a fine mesh is required.

This characteristic mesh can be obtained easily by generating a fine mesh in the areas with sharp changes in the geometry and generating a coarse mesh in the areas where the geometry is constant. The meshes obtained using the Adaptive Method assure this characteristic mesh automatically comparing with methods such as the Delaunay-Advancing front, which cannot obtain them automatically. In the case of the uniform meshes, another mesh generation procedure would be needed (remeshing) including a manual refinement in the areas of interest to obtain a mesh that would accomplish these characteristics. It would substantially increase the elapsed time spent to create the mesh. In the Figure 5 is observed how the low stresses in the dorsal endocranial area of the skull have practically the same distribution for a coarse mesh (meshes 1 and 5) than for a fine mesh (meshes 2, 3 and 4).

The Adaptive mesh better suits irregular geometries. But although the elapsed time spent in the mesh generation is higher, comparing with uniform meshes, it will be reduced in the solving procedure of the mesh (as Table 3 shows).

\subsection{Reliability of the results}

The reliability of the mesh evaluates how the result mathematically obtained by the Finite Element Method is close to the theoretical value.

Bearing in mind that the different meshes obtained from the different methodologies as well as the convergence procedure, it could be observed how these results are quite similar in most parts of the mesh. This is especially true for the lower values of von Mises stress distributed in practically the same areas have similar values. In contrast, when the values are higher the changes in the pattern are more pronounced (Fig. 3).

There are different reasons to explain this trend. Firstly, the stress gradients are better captured when using a small size element whenever this stress gradient has fast variations. And secondly, the artificial noise grows in direct relationship with the decrease of the size of the elements. The changes in the higher values can be observed in more detail in Table 3, where the higher values in the three middle lines of the Figure 1 are captured for the five different meshes.

During the iterative process of the convergence test the elapsed time spent in mesh 5 includes the mesh generation and the solution of the mesh. It is because the algorithm creates the new mesh and obtains the solution in each step. Therefore, considering that the convergence procedure is the one with the most reliable solution, the results obtained for coarse meshes are far from the converged solution more than the fine meshes. In Figure 4 it is specially shown how the distribution of the von Mises stress changes due to changes in the mesh, especially if it is a coarse or a fine mesh. Considering both elapsed times spent in creating the mesh (Table 2) and solving the mesh (Table 3), convergence procedure is the one with the lower value.

Table 3. Maximum values recorded in the 5 meshes, total deformation, Von Mises Stress in the line 1, in the line 2 and in the line 3 and Elapsed Time. *Mesh 5 includes the mesh generation and the solve Elapsed Time.

\begin{tabular}{|c|c|c|c|c|c|}
\hline \multirow[b]{2}{*}{ Case } & \multicolumn{4}{|c|}{ Maximum values recorded } & \multirow[b]{2}{*}{$\begin{array}{l}\text { Elapsed } \\
\text { Time [s] }\end{array}$} \\
\hline & $\begin{array}{c}\text { Total } \\
\text { Deformation } \\
(\mathbf{m m})\end{array}$ & $\begin{array}{c}\text { Von } \\
\text { Mises } \\
\text { Stress } 1 \\
\text { (MPa) }\end{array}$ & $\begin{array}{c}\text { Von } \\
\text { Mises } \\
\text { Stress } 2 \\
\text { (MPa) }\end{array}$ & $\begin{array}{c}\text { Von } \\
\text { Mises } \\
\text { Stress } 3 \\
\text { (MPa) }\end{array}$ & \\
\hline Mesh 1 & 2.7336 & 55.331 & 101.53 & 103.58 & 10 \\
\hline Mesh 2 & 2.9558 & 54.713 & 111.64 & 114.77 & 680 \\
\hline Mesh 3 & 2.6494 & 53.324 & 85.02 & 102.82 & 18 \\
\hline Mesh 4 & 3.0112 & 54.718 & 111.45 & 122.53 & 495 \\
\hline Mesh 5 & 2.8374 & 54.703 & 112.1 & 124.62 & $265^{*}$ \\
\hline
\end{tabular}

Furthermore, Figure 3 reveals that the deformation distribution is practically the same in the different meshes while Table 3 shows the similar pattern since the maximum deformation recorded differs little from each mesh. This robustness of the values of the deformation in front of the different meshes occurs because deformation is the direct solution of the equations system and the result obtained for a coarse mesh will be a very good approximation value. The only problems possible to appear with the results are the stresses, because they are not a direct solution of the equations system. They must be calculated from the deformation integrating the equations in the domain.

\subsection{Efficiency and time reduction in $\mathrm{FE}$ analysis}

Another important issue to point out is the design of the biomechanical study and how to solve it. One possible solution could be to reduce the mesh of the model by taking advantage of the bilateral symmetry present in the 

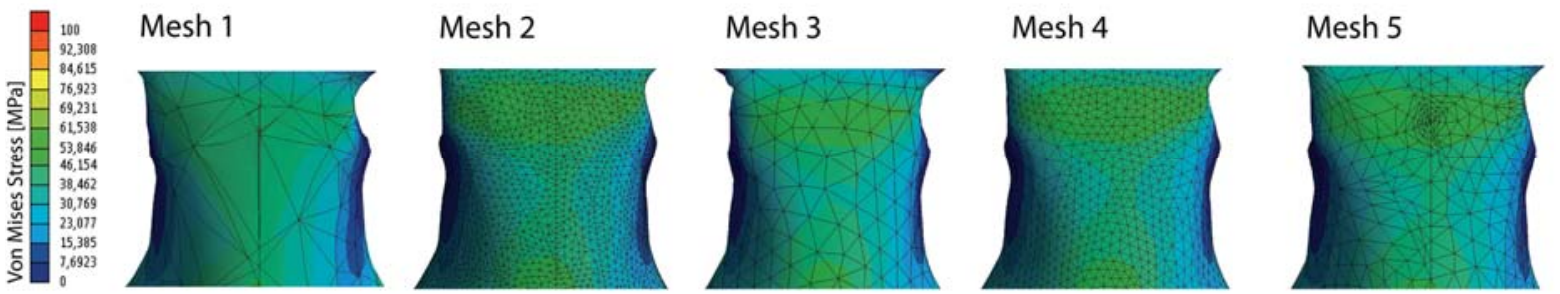

Figure 4. Von Mises Stress distribution in the frontal-parietal bones for the 5 different meshes solved. Mesh 1: Coarse Uniform; mesh 2: Fine Uniform; mesh 3: Coarse Adaptive; Mesh; mesh 4: Fine Adaptive; mesh 5: final Converged Mesh.

biological structures (see Supplementary Information for further details about symmetries). Herein, we used the bilateral symmetry of the A. davidianus skull to generate a mesh containing the half of the original domain (Fig. 5). In similar way, Wroe et al. (2007b) considered the bilateral symmetry of the skull in some of their computer simulations of hominid cranial mechanics. The mesh of their whole hominid model reached more than 3 million brick elements. However, in the case of the bilateral bite, Wroe et al. (2007b) considered the bilateral symmetry and obtained a mesh with less than 2 million elements. As a consequence of the reduction of the number of the elements, the computational time spent in performing the calculations could be reduced by half.

Otherwise, as a consequence of the mesh reduction, the mesh density may be increased obtaining greater accuracy with fewer elements than if the whole structure is modelled. This might be particularly useful if a convergence curve has to be used in the model.

Considering the above options to reduce the mesh and to increase the accuracy of the model, the first step is to check if our domain accomplishes the symmetry conditions while, the second step is to divide the domain in two symmetrical parts and finally generate the mesh. The number of nodes and elements of the new mesh would be approximately the half of the nodes and elements of the whole domain.

Finally, the last issue to take into account in order to generate an efficient mesh as suggested is the generation of a structured mesh whenever the domain allows it (see supplementary information about structured meshes). If the domain is irregular, the generation of a structured mesh could be difficult. In these cases, it is suggested to create a hybrid mesh. These types of meshes include unstructured and structured parts, and the main goal is to generate the maximum of possible parts with a structured mesh. In our case, we tested the importance of the hybrid meshes (Fig. 5): one is a hybrid mesh with a regular hexahedral mesh while another is a hybrid mesh with hexahedral and tetrahedral elements.

The best areas to be meshed as a structured mesh are the parts of the model with regular thickness. In this case we suggest focusing the efforts on giving the necessary instructions to the mesh tool of the FEA package to create a structured mesh. This will improve the quality of the mesh and the size of the storing file. Table 4 shows how the number of nodes and elements can be lower than the whole model and how the hybrid meshes can improve the quality of the mesh in spite of the high standard deviation of the second hybrid mesh (as a consequence of being together in the same mesh tetrahedron and hexahedron elements).
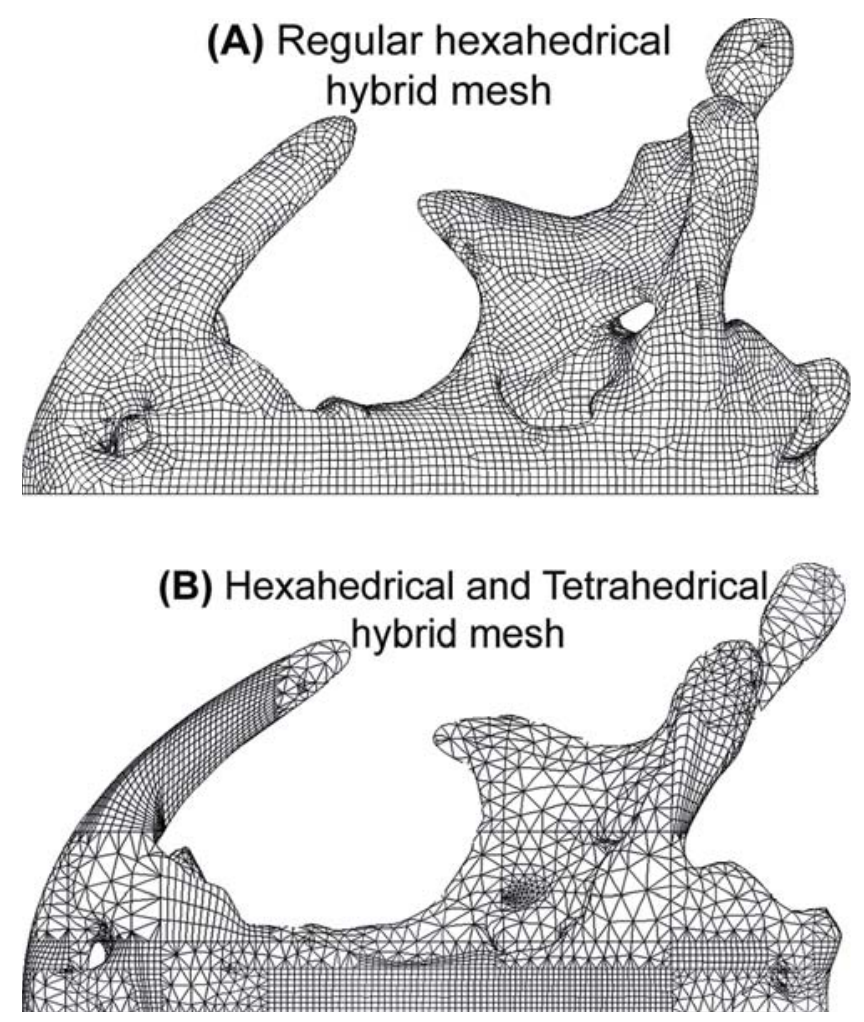

Figure 5. Half domain hybrid-structured meshes, taking advantage of the symmetric reduction. Left, mesh 6. Right, mesh 7. Note that due to the irregular geometry, mesh (a) cannot be structured in the whole domain and for this reason must be a hybrid regular mesh. 
Table 4. Nodes and elements and quality indicators of the half-domain hybrid models.

\begin{tabular}{|c|c|c|c|c|c|c|c|c|c|}
\hline \multirow[b]{2}{*}{ Case } & \multirow{2}{*}{$\begin{array}{c}\text { Mesh } \\
\text { Generation } \\
\text { Method }\end{array}$} & \multirow[b]{2}{*}{ Nodes } & \multirow{2}{*}{$\begin{array}{l}\text { Type of } \\
\text { elements }\end{array}$} & \multirow[b]{2}{*}{ Elements } & \multirow[b]{2}{*}{ Elapsed Time [s] } & \multicolumn{2}{|c|}{ Aspect ratio } & \multicolumn{2}{|c|}{ Skewness } \\
\hline & & & & & & Average & $\begin{array}{l}\text { Standard } \\
\text { deviation }\end{array}$ & Average & $\begin{array}{l}\text { Standard } \\
\text { deviation }\end{array}$ \\
\hline Mesh 6 & $\begin{array}{l}\text { Half-domain } \\
\text { hybrid mesh } 1\end{array}$ & $\begin{array}{l}\text { Only } \\
\text { Hexahedrical }\end{array}$ & 252,954 & 121,241 & 140 & 1.9743 & 0.5132 & 0.2924 & 0.1021 \\
\hline Mesh 7 & $\begin{array}{l}\text { Half-domain } \\
\text { hybrid mesh } 2\end{array}$ & $\begin{array}{l}\text { Hexahedrical } \\
\text { and Tetrahedical }\end{array}$ & 232,976 & 106,584 & 130 & 2.2131 & 1.2145 & 0.3641 & 0.5421 \\
\hline
\end{tabular}

Table 4 also shows the elapsed time in the case of Mesh 6 and Mesh 7, which is lower than in the other meshes. The elapsed time is reduced because the model has fewer nodes and because the structured parts improve the size of the storing data.

\section{CONCLUSIONS}

The mesh generation should be done with a consistent criterion to achieve an accurate final result although the overall accuracy of the results obtained in FEA will depend on the assumptions implicit in the model.

A consistent criterion may start with a first approximate analysis using a coarse mesh to explore the physical problem of the case study, and later followed by the main analysis with a suitable mesh. It is observed that the values obtained for the deformation are robust in front of the changes in the mesh and, in contrast to that, the values obtained for the stresses are absolutely dependent on the mesh. It means that the mesh will be chosen with a consistent criterion checking the stress results. For a whole mesh, it is recommended to use an adaptive mesh rather than a mesh with a uniform element size (such as those generated using the Delaunay or the Advancing front method). Firstly because the adaptive mesh has better quality indicators (skewness and aspect radio), secondly because it fits better in the irregular geometry and the stress gradients generated, and finally, because it assures more realistic results. Otherwise, it is highly recommended creating a convergence procedure when looking for a particular stress result in a specific area of the geometry. This procedure will enable us to create a final converged mesh displaying the adaptive results required without wasting computational resources in the areas with no interest. Moreover, this local analysis helps us to improve our results avoiding the artificial noise in the points where singularities appear and minimises solution time (Mesh generation and Solution of the mesh).

According to the points discussed in this paper, it can be concluded that a good, efficient mesh should meet these goals by meeting the following: a) the domain of our problem has to be represented; b) if the geometry is simple, a structured mesh is preferred over an unstructured mesh; c) good quality elements are preferred (equilateral triangles and square quadrilaterals) over lower quality elements; d) a fine mesh is recommended in the area of interest and a coarse mesh outside these areas; e) a convergence test of the mesh should be performed if it is possible in the case study, and; $f$ ) if the domain contains symmetric or asymmetric sections, consider dividing the original domain in order to create a mesh with a smaller number of nodes and elements.

\section{ACKNOWLEDGEMENTS}

Our special thanks go to: Roger Barcons for his assistance in reconstructing the Medical Image files and in creating the geometrical model of the Chinese giant Salamander (Andrias davidianus) skull; the Museu de Ciències Naturals de Barcelona for the loan of the analysed specimen; the Hospital Mútua de Terrassa for providing the facilities for performing the CT scan; Marilà Lucas (Universitat Politècnica de Catalunya) for their help in the linguistic revision and all the people of the "XI Encuentro de Jóvenes Investigadores en Paleontología", specially Carlos Martínez Pérez (University of Bristol \& Universitat de València) and Pilar Navas-Parejo (Universidad de Granada), where this work has been presented. The quality of this manuscript was greatly improved with the comments of Emily Rayfield (University of Bristol, UK) and other anonymous reviewer.

\section{REFERENCES}

Bathe, K.J. 1996. Finite Element Procedures. Prentice Hall. Bright, J.A. \& Rayfield, E.J. 2011a. Sensitivity and ex vivo validation of finite element models of the domestic pig cranium. Journal of Anatomy, 219, 456-471.

Bright, J.A. \& Rayfield, E.J. 2011b. The response of cranial biomechanical finite element models to variations in mesh density. The Anatomical Record, 294, 610-620.

Clausen, P.D., Wroe, S., McHenry, C.R., Moreno, K. \& Bourke, J. 2008. The vector of jaw muscle force a determined by computer-generated three dimensional 
simulation, A test of Greaves' model. Journal of Biomechanics, 41, 3184-3188.

Deban, S.M. \& Wake, D.B. 2000. Aquatic feeding in salamanders. In: Feeding, Form, Function, Phylogeny (ed. Schwenk, K.D). Academic Press, San Diego, California, 65-94.

Dumont, E.R., Davis, J.R., Grosse, I.R. \& Burrows, A.M. 2011. Finite element analysis of performance in the skulls of marmosets and tamarins. Journal of Anatomy, 218, 151-162.

Fletcher, T.M., Janis, C.M. \& Rayfield, E.J. 2010. Finite Element analysis of ungulate jaws, can mode of digestive physiology be determined? Palaeontologia Electronica, 13(3), 21A.

Fortuny, J., Marcé-Nogué, J., De Esteban-Trivigno, S., Gil, L. \& Galobart, Á. 2011. Temnospondyli bite club: ecomorphological patterns of the most diverse group of early tetrapods. Journal of Evolutionary Biology, 24, 2040-2054. doi:10.1111/j.1420-9101.2011.02338.x

Fortuny, J., Marcé-Nogué, J., Gil, L. \& Galobart, Á. 2012. Skull mechanics and the evolutionary patterns of the otic notch closure in capitosaurs (Amphibia: Temnospondyli). The Anatomical Record, 295, 1134-1146. doi:10.1002/ ar.22486

Grosse, I.R., Dumont, E.R., Coletta, C. \& Tolleson, A. 2007. Techniques for Modelling Muscle-Induced Forces in Finite Element Models of Skeletal Structures. The Anatomical Record, 290, 1069-1088.

Jasinoski, S.C., Rayfield, E.J. \& Chinsamy, A. 2009. Comparative Feeding Biomechanics of Lystrosaurus and the Generalized Dicynodont Oudenodon. The Anatomical Record, 292, 862-874.

Jasinoski, S.C., Rayfield, E.J. \& Chinsamy, A. 2010. Functional Implications of Dicynodont Cranial Suture Morphology. Journal of Morphology, 271, 705-728.

Kupczik, K. 2008. Virtual biomechanics, basic concepts and technical aspects of finite element analysis in vertebrate morphology. Journal of Anthropological Sciences, 86, 193-198.

Lauder, G.V. 1995. On the inference of function from structure. In: Functional Morphology in Vertebrate Paleontology (ed. Thomason, J.J.). Cambridge University Press, Cambridge, 1-18.

Lohner, R. \& Parikh, P. 1988. Three-dimensional Grid Generation by the Advancing Front Method. International Journal for Numerical Methods in Fluids, 8, 1135-1149.

McHenry, C.R., Wroe, S., Clausen, P.D., Moreno, K. \& Cunningham, E. 2007. Supermodeled sabercat, predatory behaviour in Smilodon fatalis revealed by high-resolution 3D computer simulation. Proceedings of the National Academy of Sciences, 104, 16010-16015.

Moazen, M., Curtis, N., O'Higgins, P., Evans, S.E. \& Fagan, M.J. 2009. Biomechanical assessment of evolutionary changes in the lepidosaurian skull. Proceedings of the National Academy of Sciences, 106, 8273-8277.

Morris, A. 2008. A Practical Guide to Reliable Finite Element Modelling. J. W. \& Sons Peraire, J., Vahdati, M., Morgan, K. \& Zienkiewicz, O.C. 1987. Adaptive Remeshing for Compressible Flow Computations. Journal of Computational Physics, 72, 449-466.

Peraire, J., Vahdati, M., Morgan, K. \& Zienkiewicz, O.C. 1987. Adaptive Remeshing for Compressible Flow Computations. Journal of Computational Physics, 72, 449-466.

Pierce, S.E., Angielczyk, K.D. \& Rayfield, E.J. 2009. Shape and mechanics in thalattosuchian (Crocodylomorpha) skulls, implications for feeding behaviour and niche partitioning. Journal of Anatomy, 215, 555-576.

Rayfield, E.J., Norman, D.B., Horner, C.C., Horner, J.R., Smith, P.M., Thomason, J.J. \& Upchurch, P. 2001. Cranial design and function in a large theropod dinosaur. Nature, 409, 1033-1037.

Rayfield, E.J. 2007. Finite element analysis and understanding the biomechanics and evolution of living and fossil organisms. Annual Review of Earth and Planetary Sciences, 35, 541-576.

Richmond, B.G., Wright, B.H., Grosse, I., Dechow, P.C., Ross, C.F., Spencer, M.A. \& Strait, D.S. 2005. Finite element analysis in functional morphology. The Anatomical Record, 283, 259-274.

Strait, D.S., Wang, Q., Dechow, P.C., Ross, C.F., Richmond, B.G., Spencer, M.A. \& Patel, B.A. 2005. Modelling Elastic Properties in Finite-Element Analysis, How much precision is needed to produce an accurate model?. The Anatomical Record, 283, 275-287.

Wroe S., Clausen, P., McHenry, C., Moreno, K. \& Cunningham, E. 2007a. Computer simulation of feeding behaviour in the thylacine and dingo as a novel test for convergence and niche overlap. Proceedings of the Royal Society of London Biological Sciences, 274, 2819-2828.

Wroe, S., Moreno, K., Clausen, P., McHenry, C. \& Curnoe, D. 2007b. High-resolution three-dimensional computer simulation of hominid cranial mechanics. The Anatomical Record, 290, 1248-1255. 


\section{SUPPLEMENTARY INFORMATION}

\subsection{Definition and characteristics of a mesh}

The mesh of a FEM domain is a collection of subdomains called elements interconnected between them with nodes that defines the shape of the domain where the physical problem will be solved using the finite element method. The sub-domains usually consist of triangles, quadrilaterals, tetrahedrals, hexahedrals or other simple convex polygons which simplify and discretise the geometry of the original domain.

The underlying premise of the method states that a complicated domain can be subdivided into a mesh in which the equations are approximately solved.

A problem can be solved in a two-dimensional domain or in a three-dimensional domain, however different finite elements are required as shown in Figure S1. The geometry of the original domain can be replaced by a linear element or a quadratic element depending on the decision of the mesh procedure, but in curved geometries fewer quadratic elements will be needed to approximate the geometry comparing with the number of linear elements needed to obtain the same geometric approximation.

When the domain consists of surfaces or lines, the mesh created uses shell elements or beam elements projected in a three-dimensional space, as Figure S2 shows. These elements need a different formulation to the solid elements explained above but, usually, FEM packages control its usages.

Today, the main commercial packages of FEA include several methods to automatically generate the mesh of the domain without human intervention. This improves the analysis but the mathematically complexity of the FEM will not avoid the mesh generation still having to be treated with care. It is important to find a consensus between the best mesh to be built and analysed and the limits of our computational machines.

\subsection{Mesh Generation methods}

Regardless of whether a manual or automatic mesh generation method is used, the process consists of different tasks, initial mesh generation, remeshing and refinement.

\subsubsection{Initial mesh generation}

Initial mesh (Fig. S3.1) generation requires a description of the mesh domain geometry. The domain is usually generated using CAD tools and the mesh generator will then create a mesh that covers the domain according to the mesh size requirements specified by certain control

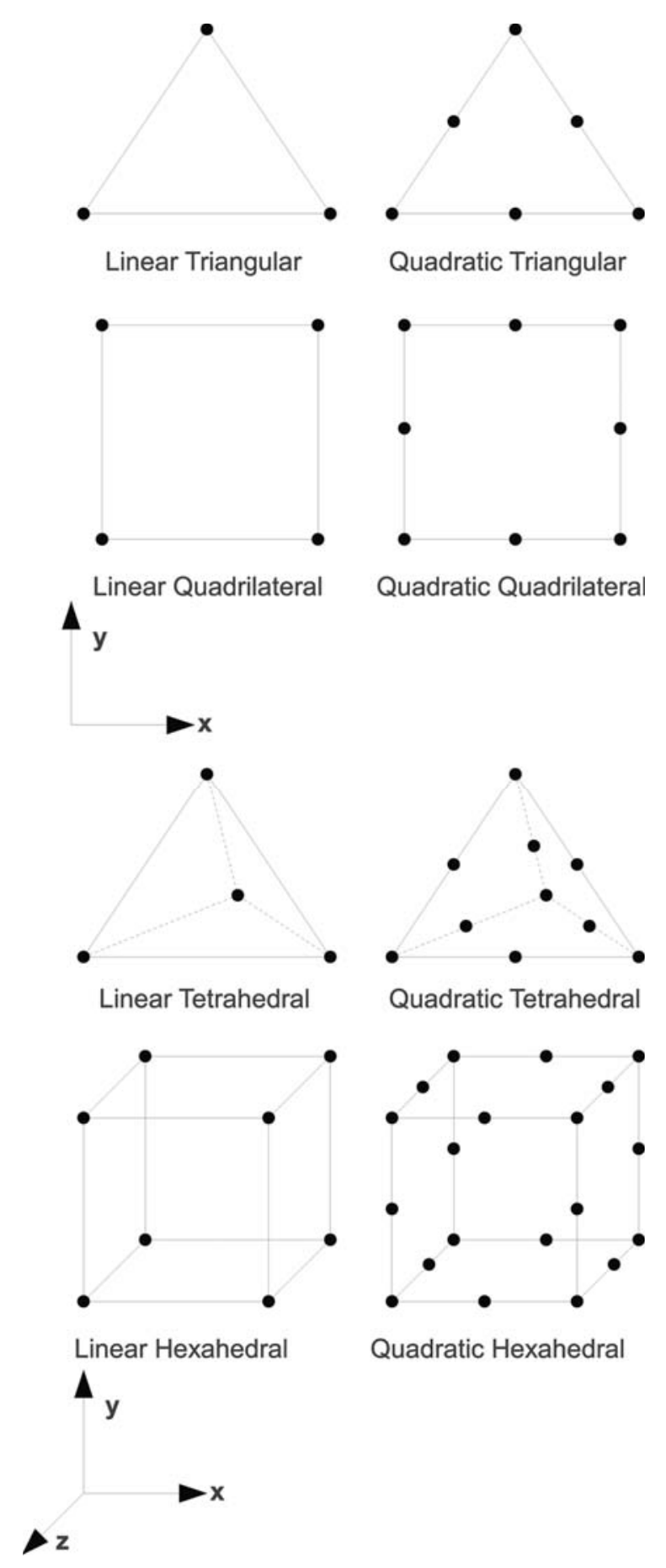

Figure S1. Type of two-dimensional and three-dimensional finite elements.

parameters. The initial mesh can be called the coarse mesh, since it may be improved by remeshing or refinement in the following tasks.

\subsubsection{Remeshing}

This is carried out when a given mesh, usually the initial or coarse mesh, no longer satisfies the established criteria 


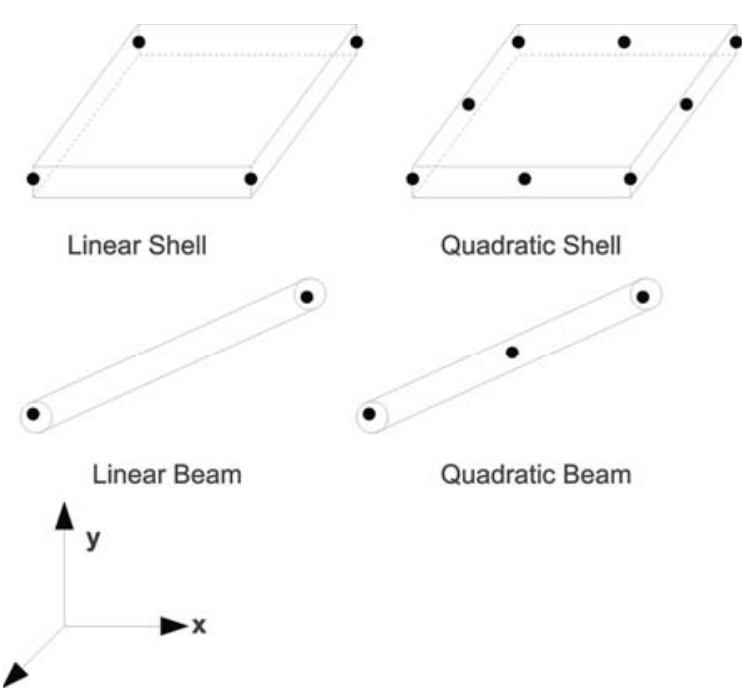

Figure S2. Type of shell and beam finite elements.

for the mesh size or element quality. Then, a new mesh is created using the initial mesh as a starting domain for the new (Fig. S3.2).

\subsubsection{Refinement}

This occurs when additional elements are formed in one or more areas of an initial mesh (Fig. S3.3).

\subsection{Types of mesh}

\subsubsection{Uniform and non-uniform mesh}

A uniform mesh consists of a mesh in which the element size is the same for all elements. A non-uniform mesh is a mesh in which the element size is different (see Fig. S4).

\subsubsection{Structured and unstructured mesh}

A structured mesh (Figs S4.1-2) consists of two sets of lines forming a regular grid. The lines in the same set do not intersect each other. These lines only intersect with other lines from other sets once generating the regular grid since the connectivity of the elements is regular. The basic methods used to generate structured meshes classified by Frey \& George (2000) are, Algebraic interpolation methods, solving partial differential equations and multiblock.

An unstructured mesh (Figs S4.3-4) occurs when it is not the result of the intersection of sets of parallel lines. There are a lot of unstructured mesh generators and the three most importants are, the advancing front method (Lohner \& Parickh, 1988), the Paving method (Blacker \&

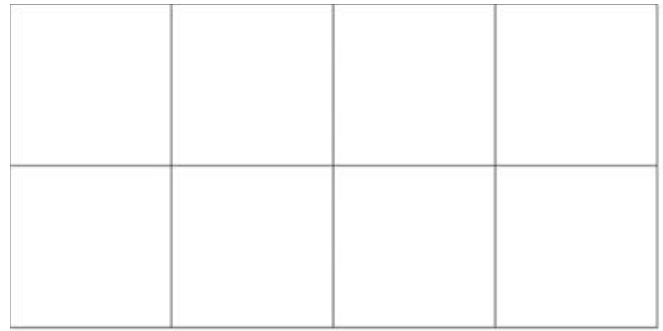

(1)

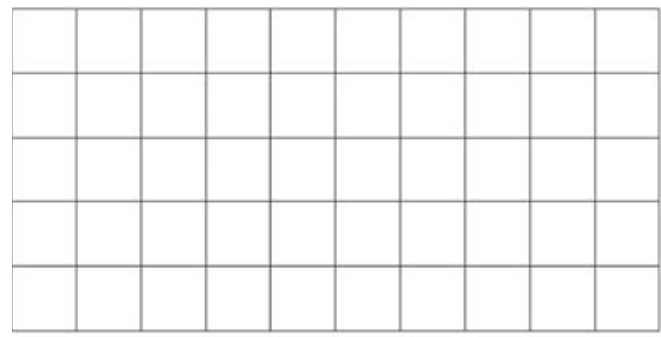

(2)

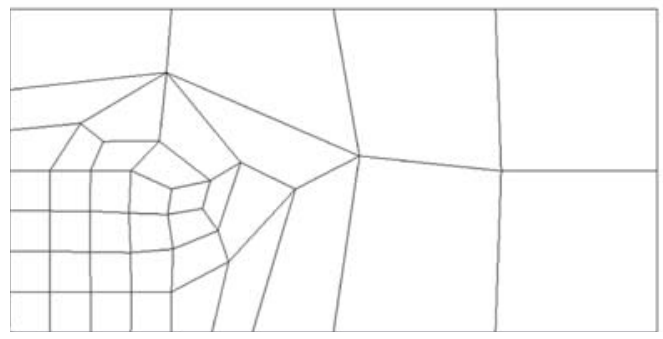

(3)

Figure S3. 1) Initial, 2) remeshed and 3) refined mesh.

Stephenson, 1991) and the Delaunay triangulation (Peraire et al., 1987).

A hybrid mesh (Fig. S4.5) consists of one part of structured mesh and another part of unstructured mesh.

\subsubsection{Advantages and disadvantages}

The automatic mesh generation method applied depends on the type of mesh. Depending on its type, one generation method will be more optimal than another. Structured meshes are generally created for quadrilateralshaped elements (quadrilaterals, hexahedrals, etc.) while unstructured meshes are generally used for triangularshaped elements (triangles, tetrahedrals, etc.).

A triangular-shaped mesh can be generated quickly, automatically, and for complicated geometries it is a shape perfectly suitable for covering the entire domain. However, for an isotropic refinement (in order to capture gradients in one direction, mesh is automatically refined in all three directions) cell counts rise rapidly and the number of elements and nodes are higher than those in a hexahedral mesh with a similar mesh density.

A quadrilateral-shaped mesh usually has fewer than half of the nodes of a tetrahedral mesh. By contrast, for arbitrary geometries, hexahedral meshing is not always suitable or may require a multi-step process (wasting a lot 


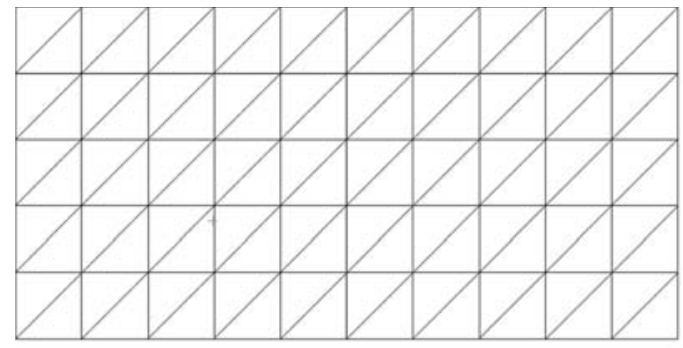

(1)

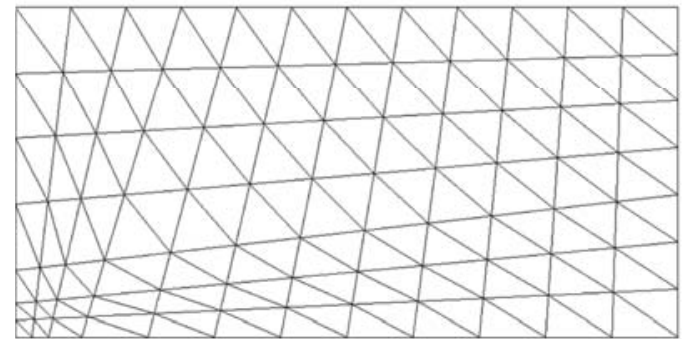

(2)

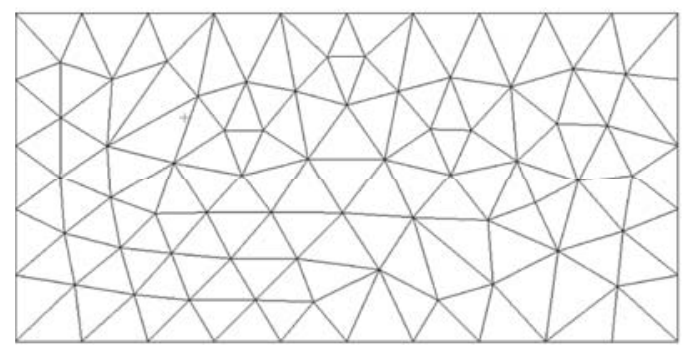

(3)

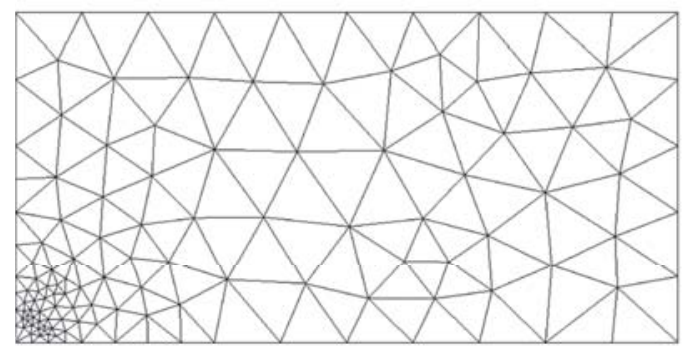

(4)

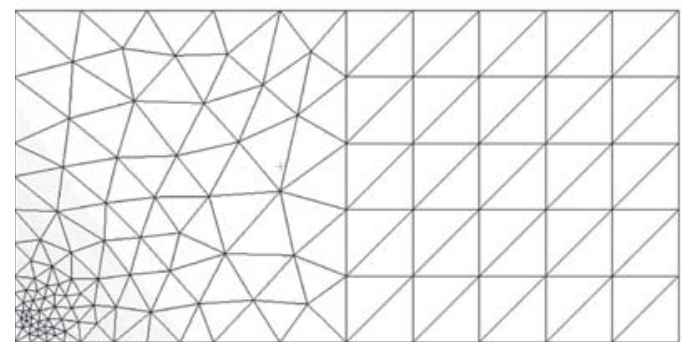

(5)

Figure S4. 1) Structured uniform, 2) structured nonuniform, 3) unstructured uniform, 4) unstructured non-uniform and 5) hybrid mesh.

of time in obtaining a good mesh), although the final mesh can yield high quality/high efficiency. In any case, the structured meshes require less memory to store information and it will be recommended to use this type of meshes at any time domain allows it. If the domain is irregular we suggest creating a hybrid mesh to take the advantage of a structured mesh in the parts which is possible and an unstructured mesh in the parts in which the complexity of the geometry cannot allow a structured mesh.

\subsection{Error analysis}

The objective of the error indicators is to evaluate how close the finite element solution is to the exact solution of the mathematical model and, therefore, whether the generated mesh is good enough to give us the right solution to the problem. The errors in a finite element analysis come from mathematical modelling, finite element formulation and model mesh. The errors from the mathematical model and the finite element formulation arise in mathematical theory and do not affect the mesh generation. The mesh error is generally caused during the transformation of the original domain (which consists of a continuum media) into a number of finite elements. These error estimates may be used as useful information in adaptively refining the mesh. In most FEA packages this process is now performed automatically by relating the error estimate to mesh size parameter, which implies that with higher error estimates, the size of the generated mesh is smaller.

\subsection{Mesh quality}

In the areas where the original domain has a high geometric complexity, the mesh elements created in the mesh generation process can become distorted and, consequently, these elements will produce poor quality indicators. Poor quality elements can lead to poor quality results or, in some cases, no results at all. In the literature, there are several parameters to assess the quality of the mesh, such as indicators for measuring an specific item or the whole shape of the mesh, Aspect Ratio, Jacobian Ratio, Warping Factor, Parallel Deviation, Maximum Angle, Skewness or Taper (see Topping et al., 2004 for more details).

These parameters can be used to construct an overall indicator of the quality of the mesh, thus obtaining the different quality indicators and limiting this value to a minimum threshold or to obtain the average rate of various quality indicators. There are indicators for all type of elements (triangular, quadrilaterals, two-dimensional and three-dimensional) and they are listed in the literature (see Topping et al., 2004 for more details).

Generally, these quality indicators are higher when the triangular elements are equilateral and the quadrilateral elements are perfectly squared. Therefore, the quality of the mesh will be better when the elements are closer related to regular shapes. When the mesh has a good quality, the error is smaller than in meshes with a poor quality due to the finite element formulation (Fig. S5).

The three most important factors affecting mesh quality are, 1) the CAD issues; 2) the mesh resolution and distribution, and; 3 ) the meshing method used to generate it. 


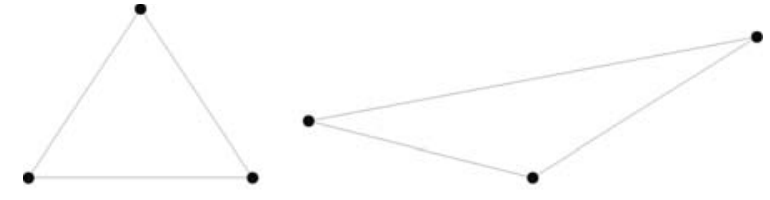

Aspect ratio $=1$

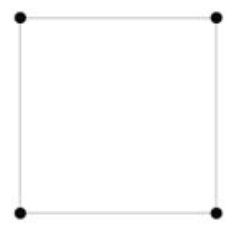

Aspect ratio $=1$

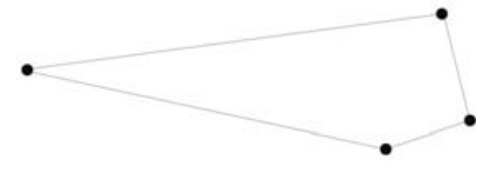

High aspect ratio

Figure S5. Aspect ratio of triangular and quadrilateral elements.

CAD issues need to be previously fixed to avoid small edges, sharp edges and faces, small gaps/passages between edges and faces and unconnected geometry entities. This can be done by simplifying the geometry, merging faces or edges, avoiding narrow faces, keeping volume gaps only where they are important, decomposing the geometry, removing unnecessary geometries or repairing the geometry. These tasks should be done before the mesh is generated.

Inappropriate mesh resolution and distribution may lead to large cell quality mesh indicators. Geometry with abrupt changes, discontinuities and/or small gaps may require higher mesh resolution and must take into account the most appropriate distribution in every case.

To sum up, inappropriate usage of Meshing Method may lead to poorer quality mesh indicators because the selection of the Meshing Method depends on the geometry and application.

\subsection{Convergence of the mesh}

The convergence of the mesh refers to the procedure to obtain sufficient smallness of the elements required in a model to ensure that the results of an analysis are not affected by changing the size of the mesh. The optimal solution would be the finest possible mesh because a finer mesh typically results in a more accurate solution. But, as a mesh is made finer, the computation time increases. The goal of a convergence study is to look for the most optimal solution which ensures the best result with the coarsest mesh possible.

\subsubsection{Convergence curve}

The convergence curve plots a critical result parameter within a specific location against some measure of mesh density or refinement iteration. At least two iterations of the solution procedure will be required to plot the curve. The convergence is achieved when the difference between the two last iterations is less than a certain error threshold (Fig. S6). The size of the mesh (of this refinement iteration) is smaller than the initial size and can be considered good enough to capture the results in the domain without the necessity of refining the mesh again.

When the refinement is applied in the convergence study, all the elements of the model should be split in all directions. However, it is not necessary to perform this refinement in the whole model because of the Saint Venant Principle. This principle states that local stresses in one specific region of a structure do not affect the stresses in the other.

This statement allows an adaptive mesh of the domain to be generated by converging the model by refining the mesh only in the regions where the convergence is applied, to retain the unrefined mesh elsewhere and to have transition regions, from coarse to fine meshes (Fig. S6).

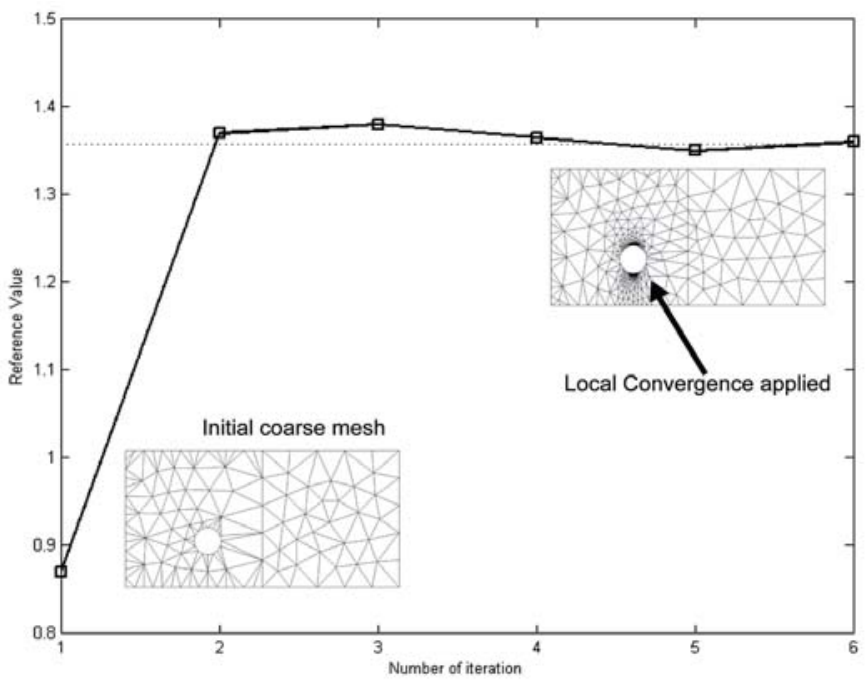

Figure S6. Convergence mesh diagram with the initial coarse mesh and the final adaptive mesh using a Convergence criterion in a specific point of the domain.

\subsubsection{Meshing strategy}

The use of local mesh refinement for a convergence study can be extended to produce accurate results in specific regions of the domain with significant implications for our purposes. Thus, an adaptive mesh could be considered, using a very fine mesh in the regions of interest, and a coarse mesh in the others regions.

The role of all elements away from the regions of interest only represents geometry and transmitting load, 
without affecting the results. In the regions of no interest, the level of the mesh is lower than for accurate result predictions and these elements can be larger and create a coarse mesh. Nevertheless, this coarse mesh should be subject to the constraints to allow both reasonable quality transitions and geometry representation and must not misrepresent the geometry.

This strategy will enable our model to find the required balance between a fine mesh in the points of interest and a coarse mesh outside, which drastically reduces the overall number of elements of the domain mesh and, consequently, the computational time.

In Figure S6, the convergence criterion is only applied in the central hole of the rectangle. We are not interested in the results of the distant regions and the mesh is only refined near the hole. This strategy is called efficiency; greater numbers of elements require more computing resource (memory /processing time). It is important to balance the faithfulness of the simulation with available resources. This strategy is better than to entire the mesh of the model with small, high quality elements in order to improve the overall accuracy in the whole domain and resulting in an inefficient strategy.

\subsubsection{Automatic convergence}

Initial refined mesh is automatically generated by most FEA packages and convergence tools are used as part of the solution process (h-adaptive method). This is done by some FEA packages to control the accuracy for selected stress results and employing an adaptive solver engine to identify and refine the model in areas that benefit from adaptive refinement The Zienkiewicz-Zhu norm (1992a, b) for stress in structural analysis is used to control the element's results and convergence whenever possible.

\subsection{Symmetric areas}

A domain is symmetrical when it is identical on either side of a dividing line or plane (Fig. S7). Along the line or plane of symmetry, boundary conditions must be applied to represent the symmetrical part. A domain is antisymmetrical when the loading of a model is oppositely balanced on either side of a dividing line or plane (Fig. S7). Boundary conditions must be applied along the line of symmetry representing the anti-symmetric part.

In this case "identical" means that four conditions are achieved, 1) the domain; 2) the boundary conditions; 3) the material properties, and; 4) the loads are symmetrically the same in each part of the domain.

If the domain of a model can be divided with natural lines of symmetry and anti-symmetry, it could be interesting to analyse only a portion of the model. This technique reduces the total number of nodes and elements of the model, reduces the time needed for computational analysis, and also the demands of the computer resources.

In this case, the construction of the model can include a symmetrical portion (half, quarter, eighth, etc.), by applying the appropriate boundary conditions. The advantages of a symmetrical and an anti-symmetrical model include the following; the processing analysis of a symmetrical portion (of a structure) is faster than if the whole structure is modelled. Furthermore, it is possible to increase the density of the symmetrical model obtaining greater accuracy and still keep a few elements, rather than if the whole structure is modelled. Therefore, symmetry and anti-symmetry approaches are good strategies to improve the efficiency of the model.

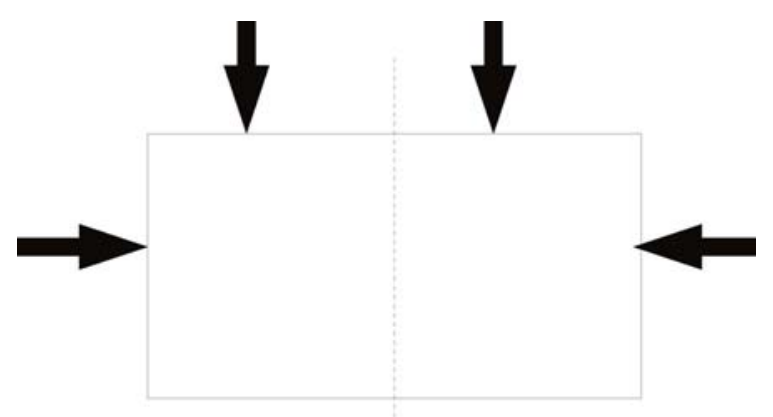

Symmetric domain

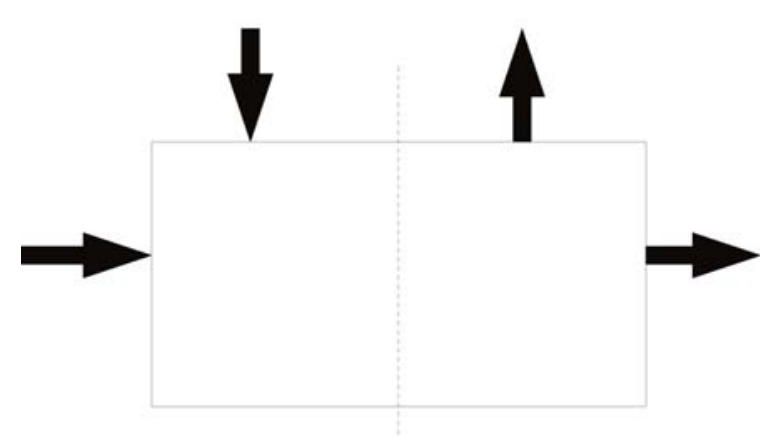

Antisymmetric domain

Figure S7. Symmetric and Antisymmetric domain.

\section{REFERENCES}

Bathe, K.J. 1996. Finite Element Procedures. Prentice Hall. Blacker, T.D. \& Stephenson, M.B. 1991. Paving, a new approach to automated quadrilateral mesh generation. International Journal for Numerical Methods in Engineering, 32, 811-847.

Frey, P.J. \& George P.L. 2000. Mesh generation, application to finite elements. Hermes Science Publishing, Oxford, England. 
Lohner, R. \& Parikh, P. 1988. Three-dimensional grid generation by the advancing front method. International Journal for Numerical Methods in Fluids, 8, 1135-1149.

Peraire, J., Vahdati, M., Morgan, K. \& Zienkiewicz, O.C. 1987. Adaptive Remeshing for Compressible Flow Computations. Journal of Computational Physics, 72, 449-466.

Topping, B.H.V., Muylle, J., Iványi, P., Putanowicz R. \& Cheng B. 2004. Finite element mesh generation. SaxeCoburg Publication.
Zienkiewicz, O.C. \& Zhu, J.Z. 1992a. Super convergent patch recovery and a posteriori error estimators. Part 1 . The recovery technique. International Journal for Numerical Methods in Engineering, 33, 1331-1364.

Zienkiewicz, O.C. \& Zhu, J.Z. 1992b. Super convergent patch recovery and a posteriori error estimators. Part 2. Error estimates and adaptiveness. International Journal for Numerical Methods in Engineering, 33, 1365-1382. 